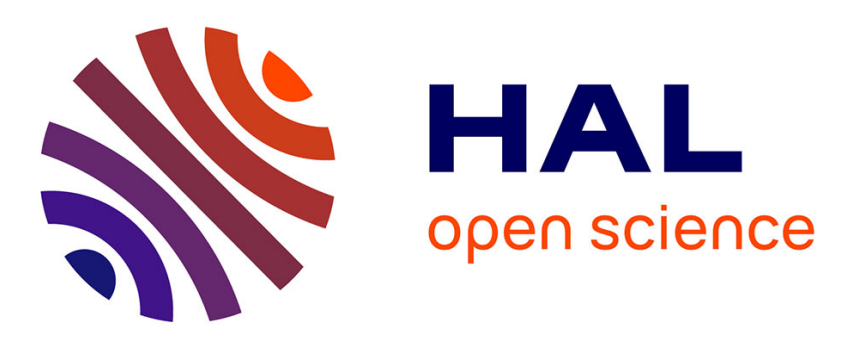

\title{
Evolution of a mushy zone in a static temperature gradient using a volume average approach
}

André B. Phillion, Miha Založnik, I. Spindler, N. Pinter, C.-A. Aledo, Georges

Salloum-Abou-Jaoude, Henri Nguyen Thi, Guillaume Reinhart, Guillaume

Boussinot, Markus Apel, et al.

\section{To cite this version:}

André B. Phillion, Miha Založnik, I. Spindler, N. Pinter, C.-A. Aledo, et al.. Evolution of a mushy zone in a static temperature gradient using a volume average approach. Acta Materialia, 2017, 141, pp.206 - 216. 10.1016/j.actamat.2017.09.011 . hal-01709331

\section{HAL Id: hal-01709331 \\ https://hal.univ-lorraine.fr/hal-01709331}

Submitted on 14 Feb 2018

HAL is a multi-disciplinary open access archive for the deposit and dissemination of scientific research documents, whether they are published or not. The documents may come from teaching and research institutions in France or abroad, or from public or private research centers.
L'archive ouverte pluridisciplinaire HAL, est destinée au dépôt et à la diffusion de documents scientifiques de niveau recherche, publiés ou non, émanant des établissements d'enseignement et de recherche français ou étrangers, des laboratoires publics ou privés. 


\title{
Evolution of a mushy zone in a static temperature gradient using a volume average approach ${ }^{\text {* }}$
}

\author{
A.B. Phillion ${ }^{\mathrm{a}, *}$, M. Založnik ${ }^{\mathrm{b}}$, I. Spindler ${ }^{\mathrm{c}}$, N. Pinter ${ }^{\mathrm{c}}$, C.-A. Aledo ${ }^{\mathrm{c}}$, \\ G. Salloum-Abou-Jaoude ${ }^{\mathrm{d}}$, H. Nguyen Thi ${ }^{\mathrm{d}}$, G. Reinhart ${ }^{\mathrm{d}}$, G. Boussinot ${ }^{\mathrm{e}}$, \\ M. Apel ${ }^{\mathrm{e}}$ H. Combeau ${ }^{\mathrm{b}}$ \\ ${ }^{a}$ Department of Materials Science and Engineering, McMaster University, Hamilton, \\ Canada \\ ${ }^{b}$ Institut Jean Lamour, CNRS - Université de Lorraine, Nancy, France \\ ${ }^{c}$ École nationale supérieure des mines de Nancy, Université de Lorraine, Nancy, France \\ ${ }^{d}$ IM2NP, CNRS \& Universités d'Aix-Marseille et de Toulon, France \\ ${ }^{e}$ Access e. V., Aachen, Germany
}

\begin{abstract}
A volume average model to study the transition of a semi-solid mushy zone to a planar solid/liquid interface in a static temperature gradient is presented. This model simulates the principal phenomena governing mushy zone dynamics including solute diffusion in the interdendritic and bulk liquids, migration of both the solid-liquid interface and the mushy-liquid boundary at the bottom and top of the mushy zone, and solidification.

The motion of the solid-liquid interface is determined analytically by performing a microscopic solute balance between the solid and mushy zones. The motion of the mushy-liquid boundary is more complex as it consists of a transition between the mushy and bulk liquid zones with rapidly changing macroscopic properties. In order to simulate this motion, a control volume characterized by continuity in the solute concentration and a jump in both the liquid fraction and the solute concentration gradient was developed.

The volume average model has been validated by comparison against prior in-situ X-ray radiography measurements [1], and phase-field simulations [2] of the mushy-to-planar transition in an $\mathrm{Al}-\mathrm{Cu}$ alloy. A very good similarity was achieved between the observed experimental and phase-field dynamics with this new model even though the described system was only one-dimensional. However, an augmentation of the solute diffusion coefficient in the bulk liquid was required in order to mimic the convective solute transport occurring in the in situ X-ray study. This new model will be useful for simulating a wide range of natural and engineering processes.
\end{abstract}

Keywords: Solidification, Volume averaging method, Phase field method, Synchrotron x-ray radiography, Temperature gradient zone melting 


\section{Introduction}

At the scale of microstructure, alloys either solidify via continuous cooling or in a static temperature gradient. The first case is well known, representing most industrial and physical processes where materials solidify in a certain temperature interval when the melt is cooled down. The second case, while being less common, nevertheless represents an important process occurring in diverse situations such as at the surface of an exoplanet [3], in a lava lake [4], or in the concrete-uranium mixture known as corium that forms during nuclear accidents [5]. Solidification in a static temperature gradient occurs because the transient heat transfer initially creating the mushy zone has reached steady state conditions. In the case of nuclear accidents, for example, steady state heat transfer is established after a certain time period whereby the heat released by the decaying uranium is balanced by the heat absorbed by the ground [6]. The result is that an initially dendritic structure will evolve into a planar interface and thus solidify in a manner similar to temperature gradient zone melting (TGZM) [7, 8, 9]. If such a transition occurs, it significantly modifies the heat transfer through the system and thus affects the survival and integrity of the various reactor structures [5].

Solidification within a static temperature gradient has been the focus of a number of recent studies. Fischer et al. [10] investigated mushy zone dynamics in a temperature gradient for $\mathrm{Al}-\mathrm{Cu}$ alloys, extracting local mean composition and solid fraction data from the experimental results. D. Liu et al. [11] evaluated the influence of thermal stabilization on the solute concentration within the melt in both directionally solidified $\mathrm{Al}-\mathrm{Ni}$ and $\mathrm{Al}-\mathrm{Cu}$ systems, illustrating the contrasting effects of $\mathrm{Ni}$ and $\mathrm{Cu}$ elements on segregation. T. Liu et al. [12] performed a similar study on a Ti-43Al-3Si alloy in order to investigate mechanisms for obtaining a lamellar microstructure. Recently, Salloum-Abou-Jaoude et al. [1] carried out a series of X-ray radiography experiments at the European Synchrotron Radiation Facility (ESRF) to directly observe the evolution of an Al-Cu mushy zone in a static temperature gradient of approx. $2700 \mathrm{~K} / \mathrm{m}$. Through this work they showed that it was the competition between diffusion processes in the bulk and in the mushy zone that led to the planar interface.

Concurrent to experiments, mathematical models have been proposed with the objective of providing a theoretical framework of this process and a quantitative prediction of its dynamics. Combeau et al. [5] approximated solidification within a static temperature gradient using an analytical approach in order to determine the time delay required for the planar interface to fully develop in

\footnotetext{
This is a post-peer-review, pre-copyedit version of an article published in Acta Materialia: A.B. Phillion, M. Založnik, I. Spindler, N. Pinter, C.-A. Aledo, G. Salloum-Abou-Jaoude, H. Nguyen-Thi, G. Reinhart, G. Boussinot, M. Apel, H. Combeau, Evolution of a mushy zone in a static temperature gradient using a volume average approach, Acta Mater. 141 (2017) 206-216. The final authenticated version is available online at: http://dx.doi.org/10.1016/ j.actamat.2017.09.011.

*Corresponding author

Email address: andre.phillion@mcmaster.ca (A.B. Phillion)
} 


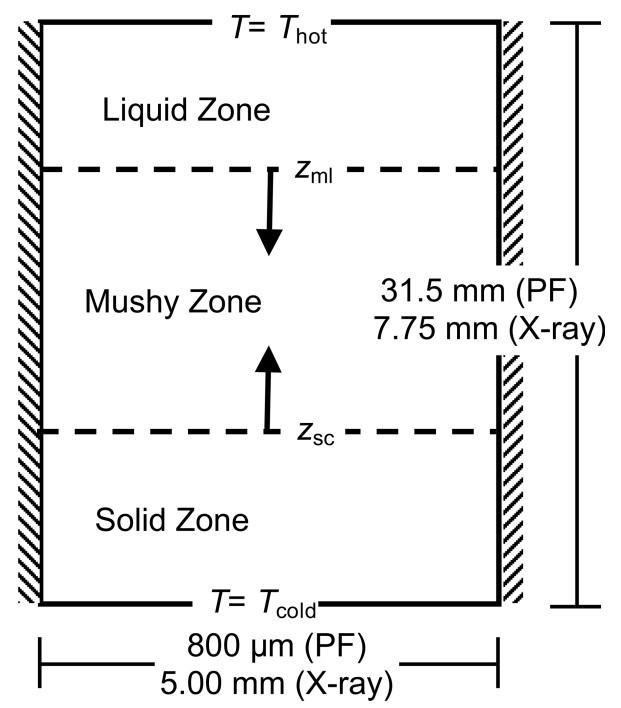

Figure 1: Schematic of the volume average model domain showing the three regions of interest (solid, liquid, mushy), along with the two mobile interfaces (solid-crust $\left(z_{s c}\right)$ and mushy-liquid $\left.\left(z_{\mathrm{ml}}\right)\right)$. The dimensions refer to the prior X-ray radiography experiments [1] and phase field [2] simulations used for comparison purposes.

corium alloys of different silica composition. They showed that the transition can follow two regimes, depending on the partition coefficient of the alloy. However, this model did not accurately consider the motion of the two boundaries between the solid, mushy, and liquid zones. Gewecke and Schulze [13] presented a similar model to study the evolution of a mushy zone cooled from below. This model included a description of the migration of the solid-mushy and mushyliquid boundaries, coupled to the diffusion in the bulk liquid. However, it was only suitable for alloys containing perfect immiscibility, i.e. the partition coefficient $k=0$. Further, as shown in the present paper, the treatment of the mushy-liquid boundary as a sharp interface is not necessarily justified. Recently, Boussinot and Apel [2] studied the complete solidification of an Al-Cu mushy zone in a static and unidirectional thermal gradient of $10^{4} \mathrm{~K} / \mathrm{m}$ by directly simulating microstructure evolution using the phase field method. This work showed clearly that liquid domains migrate toward the bulk liquid, forming a liquid channel that decreases in length due to the same process as TGZM. However, the computation cost of spatially resolved phase-field simulations limits the accessible parameter range, in particular alloy composition and thermal gradients that correspond to the necessary domain size to fully represent the mushy zone. In addition, resolidification is a slow process compared to primary solidification and thus requires the simulation of longer time periods. The phase-field computation time for the $2 \mathrm{D}$ simulations used as benchmark data in this paper was of the order of two months.

In this paper, a volume average model is proposed to study solidification 
within a static temperature gradient. A schematic of the simulation domain showing the model's three zones (solid, mushy, and liquid), the two mobile interfaces (solid crust $\left(z_{\mathrm{sc}}\right)$ and mushy-liquid $\left(z_{\mathrm{ml}}\right)$ ), along with relevant dimensions is given in Fig. 1. The new model includes solute diffusion in the mushy zone as well as in the liquid zone ahead of $z_{\mathrm{ml}}$, and takes into account the initial undercooling of the dendrite tip. The advantage of the volume average method over phase field is the significant reduction in computational time, and the corresponding ability to simulate low thermal gradients that match laboratory and industrial solidification processes. However, the description of the transition region between the mushy and liquid zones must be carefully considered with the use of averaged quantities. Prior volume average models of solidification [14] focused on the case of continuous cooling, and used a growth kinetics law for a paraboloid dendrite tip to track the dendritic front. In the case of a static temperature gradient, the shape of the solid grains is transitioning from dendritic to planar. As a dendrite-tip model cannot handle such a transition, the motion of $z_{\mathrm{ml}}$ needs to be considered at the macroscale where only the primary variables, namely the fraction solid and the solute concentration, are evaluated. Thus, special interfacial conditions are needed to describe the boundary between the two zones. First, we present the volume average model, derive the interfacial conditions and clearly show all assumptions taken. We then carefully verify these assumptions by comparison against volume average results of phase-field simulations [2]. Finally, the model is compared against prior experimental results [1] and phase field studies [2] in order to demonstrate the applicability of the volume average approach.

\section{Prior Experimental and Numerical Work}

\subsection{Experimental}

An example sequence of synchrotron X-ray radiographs taken from the work of Salloum-Abou-Jaoude et al. [1], who observed solidification of an Al-4wt.\%Cu alloy sample $5 \times 37 \mathrm{~mm}$ and $200 \mu \mathrm{m}$ in thickness in a static temperature gradient, is given in Fig. 2. Due to macrosegregation effects, caused by repeated melting and discussed below, the initial composition of the melt was actually Al-5wt.\%Cu. The initial columnar dendritic structure, shown in Fig. 2(a), was obtained by directionally solidifying the sample in a Bridgman furnace. Once the mushy zone height reached approximately two-thirds of the X-ray field of view, the cooling was stopped, resulting in a static temperature gradient, thus initiating the holding stage. The full procedure is described in [1]. As can be seen, during the holding stage the mushy zone evolved over time into a planar solid-liquid interface (Fig. 2(b,c,d)). This experiment showed that the evolution of the mushy zone can be characterized by two phenomena: an advance of the solid crust, $z_{\mathrm{sc}}$, and a retreat of the mushy-liquid boundary, $z_{\mathrm{ml}}$. Specifically, $z_{\mathrm{ml}}$ retreats with an initially high velocity that is gradually reduced, while $z_{\mathrm{sc}}$ advances until the two interfaces merge. From the collected data, the evolution in solute composition and its gradient both in the liquid and mushy zones 

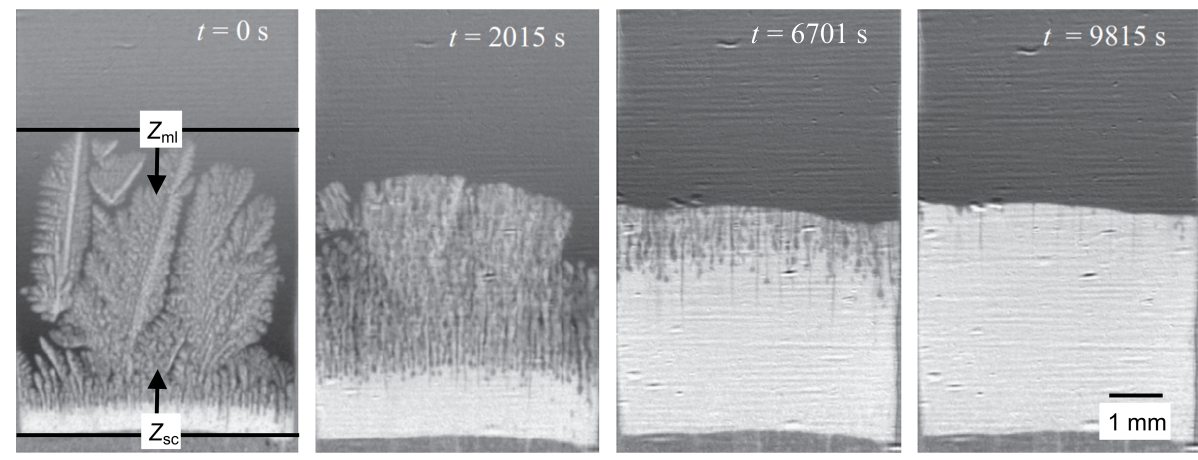

Figure 2: ESRF X-ray radiography experiment [1] to observe Al-5wt.\%Cu solidifying in a static temperature gradient. The images represent $0,2015,6701$, and $9815 \mathrm{~s}$ after the start of the hold period.

were measured and the evolution of the liquid fraction in the mushy zone was estimated.

A sequence of four experiments was performed at the ESRF. In each run, the solid structure was first melted to obtain an initial planar interface in a gradient of $2000-3000 \mathrm{~K} / \mathrm{m}$ at the bottom of the field of view. Then the hot zone was cooled at a constant cooling rate while maintaining the cold-zone temperature constant (power-down method). This triggered the growth of columnar dendrites from the initially planar interface. After the subsequent holding stage the procedure was repeated. The phenomena observed during the holding stage and their dynamics were similar in the four experiments $[1,15]$. The duration of the solidification stage was of the order of $15 \mathrm{~min}$ and that of the holding stage was of the order of $2 \mathrm{~h}$. A post-mortem composition analysis of the sample by wavelength dispersive spectroscopy (WDS) was made after the last experiment in order to check whether the repeated melting and solidification of the sample led to macrosegregation of copper in the liquid. The calibration of the radiograph grey levels to the WDS measurements showed the initial concentration of copper in the liquid in the last experiment was $5 \mathrm{wt} . \%$. This solute concentration is thus used as the initial value in the comparison volume average simulations presented in this paper.

\subsection{Phase Field Simulation}

The 2D phase field simulation of Boussinot and Apel [2] that predicts the evolution of an $\mathrm{Al}-4 \mathrm{wt} . \% \mathrm{Cu}$ alloy from a single dendrite to a planar solid-liquid interface in a static temperature gradient is given in Fig. 3 along with the supplementary movie S1. The simulation domain was $0.8 \times 31.5 \mathrm{~mm}$, which included a 2D domain $10.5 \mathrm{~mm}$ in height and a $1 \mathrm{D}$ extension of $21 \mathrm{~mm}$ to solve the farfield $\mathrm{Cu}$ concentration in the bulk liquid; only a $2 \mathrm{~mm}$ portion encompassing the dendrite tips is shown in Fig. 3. Both the static temperature gradient and the growth speed of the primary dendrite tips during the solidification stage were around 4.5 times higher than in the experiments of Salloum-Abou-Jaoude. As 
can be seen, the simulation results match qualitatively the observations from Fig. 2, however, additional details regarding the transformation from a dendritic structure to a planar interface are revealed. First, comparing Fig. 3(a) and (b), it can be seen that the position of $z_{\mathrm{ml}}$ actually continues to advance over the first $100 \mathrm{~s}$ of the holding stage because of the "inertia" effect, i. a. the relaxation of the parabolic solutal pile-up around the dendrite tip, following the sudden stopping of the cooling. This was not observed in the ESRF experiments; possibly the phenomenon was weaker because of a smaller difference in the solute concentration gradient in the mushy and in the liquid zones. Then, $z_{\mathrm{ml}}$ retreats because of a flux of $\mathrm{Cu}$ towards the liquid zone, Fig. 3(c), before reaching a minimum and slowly advancing, Fig. 3(d). Second, while contour lines of solute concentration deep in the mushy zone are horizontal, matching the thermal gradient, the contour lines near $z_{\mathrm{ml}}$ instead follow the shape of the dendrite tips. This is most evident in Fig. 3(a) but can be seen in all four images. The rate of phase change deep in the mushy zone is slow, therefore the liquid composition is very close to the thermodynamic equilibrium imposed by the solid-liquid interfaces and follows the imposed thermal field. The situation at the tips during the solidification stage and during the first $1000 \mathrm{~s}$ of the holding stage is different. The rate of solidification/melting, causing significant rejection of solute/solvent by the solid, is fast compared to the solute diffusion rate and therefore this region is out-of-equilibrium. It is this non-equilibrium state that provides the driving force for the retreat of $z_{m l}$. Third, in Fig. 3(d), a long liquid channel parallel to the thermal gradient is observed. This channel is similar to the channel structures observed in the experiment and forms due to processes similar to TGZM. The channel disappears at longer times.

\section{Volume Average Model of the Mushy Zone}

The 1D volume average model proposed in this study describes the evolution of a mushy zone in a static temperature gradient from a columnar dendritic microstructure to a planar interface.

\subsection{Initial conditions}

It is assumed that the initial mushy zone between solid and liquid zones formed during steady-state directional solidification in a fixed temperature gra-

dient. The initial position of the solid crust, $z_{\mathrm{sc}}^{0}$, can be given by the solidus or the eutectic isotherm or another phenomenon depending on the solidification conditions. This will be discussed in more detail later on. The initial position of the mushy-liquid boundary, $z_{\mathrm{ml}}^{0}$, is defined by the position of the undercooled primary dendrite tips. The constitutional undercooling of the tips, $\Delta T_{c}$, can be calculated using the KGT model [16]. Then, the temperature at the initial position of the mushy-liquid boundary is given by

$$
T\left(z_{\mathrm{ml}}^{0}\right)=T_{m}+m \cdot C_{0}+\Delta T_{c}
$$

where $T_{m}$ is the melting point of the pure material, $m$ is the linearized slope of the liquidus line from the phase diagram, and $C_{0}$ is the bulk alloy composition. 


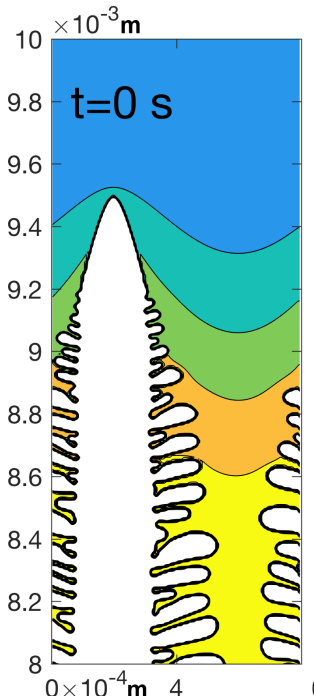

(a)

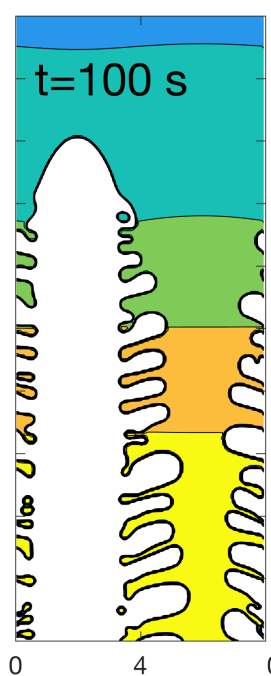

(b)

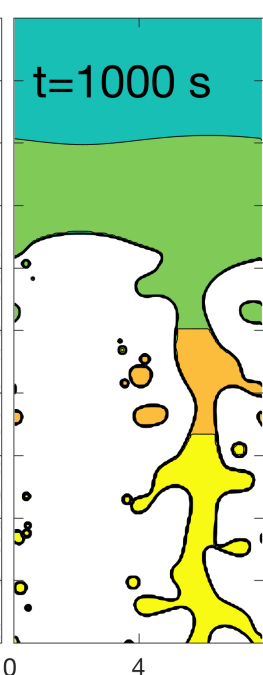

(c)

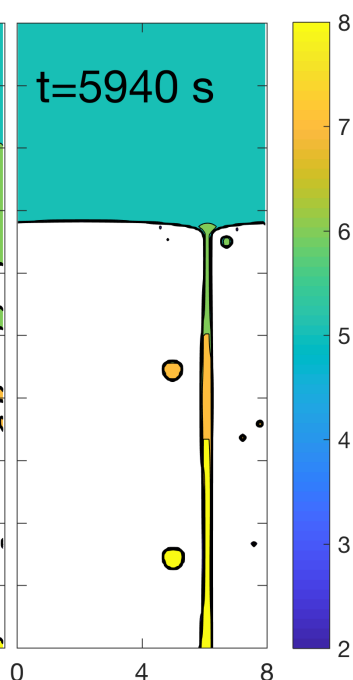

(d)

Figure 3: Phase field simulation of $\mathrm{Al}-4 \mathrm{wt} . \% \mathrm{Cu}$ solidifying in a static temperature gradient [2] The images show contours of $\mathrm{Cu}$ concentration at $\mathrm{t}=0,100,1000$, and $5915 \mathrm{~s}$ after the start of the hold period (see also accompanying video S1). The image dimensions given on the left and bottom sides of the figure are in units of metres, and the concentration legend on the right side of the figure has unit of wt.\%

As a static temperature gradient is assumed, the temperature field is stationary and does not change with time. This temperature field is given by

$$
T(z)=T\left(z_{\mathrm{ml}}^{0}\right)+G_{t h} \cdot\left(z-z_{\mathrm{ml}}^{0}\right),
$$

where $G_{t h}$ is the temperature gradient. It is also assumed that the mushy zone behind the primary dendrite tips is locally in thermodynamic equilibrium. The corresponding solute concentration field in the liquid, within the mushy zone, is thus also stationary and is given by

$$
C_{\mathrm{l}}^{\mathrm{mz}}(z)=C_{\mathrm{l}}^{0}\left(z_{\mathrm{ml}}^{0}\right)+\frac{G_{t h} \cdot\left(z-z_{\mathrm{ml}}^{0}\right)}{m},
$$

since the composition gradient in the mushy zone is directly proportional to the temperature gradient by $1 / m$. The term $C_{1}^{0}\left(z_{\mathrm{ml}}^{0}\right)=\left(T\left(z_{\mathrm{ml}}^{0}\right)-T_{m}\right) / m$ is the initial liquid composition at the mushy-liquid boundary, which will be different than $C_{0}$ because of the constitutional undercooling related to the dendrite tip growth kinetics.

The initial solute composition in the liquid zone is given by the steady-state solution of a diffusion field ahead of a boundary at $C_{l}^{0}$,

$$
C_{1}^{\mathrm{l}, 0}(z)=C_{0}+\left(C_{\mathrm{l}}^{0}\left(z_{\mathrm{ml}}^{0}\right)-C_{0}\right) \cdot \exp \left(-\frac{\left(z-z_{\mathrm{ml}}^{0}\right)}{L}\right)
$$


where $L=D_{1} / V_{\text {tip }}$ is the diffusion length, i.e. the ratio of the solute diffusion coefficient in the liquid, $D_{1}$, and the dendrite growth velocity, $V_{\text {tip }}$, at the instant the temperature field is frozen.

Finally, the initial liquid fraction field in the mushy zone is determined using the lever rule,

$$
g_{1}^{0}(z)=\frac{C_{0}-k \cdot C_{1}^{\mathrm{mz}, 0}(z)}{(1-k) \cdot C_{1}^{\mathrm{mz}, 0}(z)},
$$

where $g_{1}$ is the liquid fraction, $k$ is the partition coefficient, and the initial solute composition in the solid is equal to $k \cdot C_{1}^{\mathrm{mz}}$ at the initial timestep. Because of the constitutional undercooling of the dendrite tips, the position of $z_{\mathrm{ml}}$ is below the liquidus temperature of the alloy. Thus, in the mushy zone, the maximum liquid fraction is $\approx 0.93$.

\subsection{Governing Equations}

Equations (4) and (5) describe the initial state of the solute fields in the mushy and liquid zones and the liquid fraction field in the mushy zone. The model then predicts the evolution of these quantities with time using the governing equations described below. The evolution in liquid fraction at a given position is coupled to the solute transport, which is assumed to be by diffusion only. The governing equation for this problem is found by expressing the volume average solute conservation for the liquid phase, within the mushy zone, assuming lever rule conditions [5],

$$
\frac{\partial\left(g_{1} \cdot C_{1}^{\mathrm{mz}}\right)}{\partial t}=D^{\mathrm{mz}} \cdot \frac{\partial}{\partial z}\left(g_{1} \cdot \frac{\partial C_{1}^{\mathrm{mz}}}{\partial z}\right)+k \cdot C_{1}^{\mathrm{mz}} \cdot \frac{\partial g_{1}}{\partial t}-\left(1-g_{1}\right) \cdot k \cdot \frac{\partial C_{1}^{\mathrm{mz}}}{\partial t} .
$$

The first term on the r.h.s. of Eq. (6) describes the diffusion at the scale of the mushy zone. The second and the third term account for the exchange of solute at the local scale due to the phase change and due to the fast diffusion at the local scale in both phases, resulting in lever rule solidification. $D^{\mathrm{mz}}$ is the solute diffusion coefficient in the liquid phase within the mushy zone. Accounting for the assumption of a frozen temperature gradient which also implies $\frac{\partial C_{1}^{\mathrm{mz}}}{\partial t}=0$, Eq. (3) is substituted into Eq. (6), giving the equation for the evolution of the liquid fraction,

$$
(1-k) \cdot C_{\mathrm{l}}^{\mathrm{mz}}(z) \cdot \frac{\partial g_{1}}{\partial t}=D^{\mathrm{mz}} \cdot \frac{G_{t h}}{m} \cdot \frac{\partial g_{1}}{\partial z} .
$$

Thus, the mushy zone can be completely described by the liquid fraction field and the position of the boundaries $z_{\mathrm{ml}}$ and $z_{\mathrm{sc}}$, since $C_{1}^{\mathrm{mz}}$ is given by Eq. 3 .

In the liquid zone, the solute concentration field is given by Fick's second law,

$$
\frac{\partial C_{1}^{\mathrm{lz}}}{\partial t}=D^{\mathrm{lz}} \cdot \frac{\partial^{2} C_{1}^{\mathrm{lz}}}{\partial z^{2}}
$$

where $D^{\mathrm{lz}}$ is the solute diffusion coefficient in the liquid zone. Theoretically, the solute diffusion coefficient in the mushy and liquid zones should be nearly equal since the temperatures in these regions are similar. The need for using different diffusion coefficients will be discussed in Section 5 . 


\subsection{Boundary Conditions}

The simulation domain is considered closed, and thus solute does not leave the top of the liquid zone, nor the bottom of the solid zone, i.e. $\partial C / \partial z=0$. The boundary conditions $z_{\mathrm{ml}}$ and $z_{\mathrm{sc}}$ are key to solving this problem. Motion of $z_{\mathrm{sc}}$ is given by the migration of the solid-liquid interface at the bottom of the mushy zone. The solute diffusion at this interface is essentially one-dimensional and thus the migration of $z_{\mathrm{sc}}$ is given by a solute mass balance,

$$
(1-k) \cdot C_{\mathrm{l}}^{\mathrm{mz}}\left(z_{\mathrm{sc}}\right) \cdot v_{\mathrm{sc}}\left(z_{\mathrm{sc}}\right)=-D^{\mathrm{mz}} \cdot \frac{G_{t h}}{m},
$$

where $v_{\mathrm{sc}}$ is the interface velocity. In this balance it is assumed that the diffusion in the solid is nil and that the concentration gradient in the liquid perpendicular to the interface is $G_{t h} / m$. Note that this balance is not a balance at the macroscopic solid-mushy boundary, formulated in terms of volume averaged quantities, but a local solute balance at the solid-liquid interface, formulated in terms of local quantities. It includes the assumption that on the local (microscopic) scale the solute concentration within the liquid varies in the same way as on the macroscopic scale, i.e. following Eq. (3). This assumption will be verified later on. Eq. (9) is a first-order differential equation that has an analytical solution. The relation for $v_{\mathrm{sc}}$ is obtained by incorporating Eq. (3) into Eq. (9) and setting $z_{\mathrm{sc}}^{0}=0$,

$$
v_{\mathrm{sc}}\left(z_{\mathrm{sc}}\right)=\frac{D^{\mathrm{mz}}}{(1-k)\left(\frac{T_{m}-T\left(z_{\mathrm{sc}}^{0}\right)}{G_{t h}}-z_{\mathrm{sc}}\right)} .
$$

This equation shows that the velocity increases with the advancement of the interface in the temperature gradient. The reason is that the solute $(1-k)$. $C_{\mathrm{l}}\left(z_{\mathrm{sc}}\right)$ rejected at the interface actually decreases with increasing temperature but is evacuated by diffusion through a solute concentration gradient, $G_{t h} / m$ that is constant. By integrating Eq. (10), the equation governing the evolution of $z_{\mathrm{sc}}$ is obtained,

$$
\left(\frac{T_{m}-T\left(z_{\mathrm{sc}}^{0}\right)}{G_{t h}}\right) \cdot z_{\mathrm{sc}}(t)-\frac{z_{\mathrm{sc}}(t)^{2}}{2}=\left(\frac{D^{\mathrm{mz}}}{1-k}\right) \cdot t .
$$

The more complex boundary is between the mushy and liquid zones. This transition is more difficult to define; it does not consist of a sharp physical interface like $z_{\mathrm{sc}}$ but rather of a transition between the fully liquid zone and the mushy zone. The shape of the solid-liquid interface, the local solute concentration field and their evolution with time are all complex and evolve in two dimensions. It is therefore not possible to introduce a local description of microscopic interface conditions into the model, as has been carried out for the solid-mushy interface. The mushy-liquid boundary needs to be considered as a macroscopic interface and its description requires a proper application of the volume average method. The use of volume averaging is restricted to media 


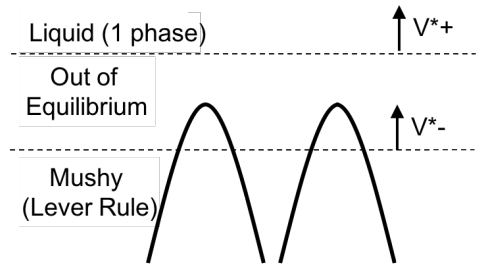

Figure 4: Schematic of the mushy / liquid interface showing the regions that are in equilibrium (Liquid, Mushy), and out-of equilibrium (Dendrite tips).

where the length scales across which macroscopic inhomogeneities are encountered are much larger than the size of the averaging volume. At the mushy-liquid boundary, the condition for the validity of the averaging procedure is thus not necessarily satisfied. In such cases, where macroscopic boundaries between two regions are characterized by rapid changes of macroscopic properties, they require a special treatment. Such interfaces must be treated as sharp surfaces where the averaged macroscopic quantities can experience a jump. Constitutive relations that describe the relationships of the properties of both domains to that of the interface must be provided. A general framework for the derivation of such relations has been developed by Hassanizadeh and Gray [17].

The solute mass balance for the boundary region is established on a control volume of finite thickness, shown in Fig. 4. The lower (-) and upper (+) edges of the control volume are in the well-defined mushy and liquid region, respectively, and move at the same rate, i.e. $v_{\mathrm{ml}}^{+}=v_{\mathrm{ml}}^{-}=v_{\mathrm{ml}}$. A balance of the total solute mass in both phases for this control volume is written as

$\epsilon_{\mathrm{ml}} \frac{\partial\langle C\rangle_{\mathrm{ml}}}{\partial t}=v_{\mathrm{ml}} \bar{C}_{1}^{+}-v_{\mathrm{ml}} \bar{g}_{1}^{-}{\overline{C_{1}}}_{1}^{-}-v_{\mathrm{ml}}\left(1-\bar{g}_{1}^{-}\right){\overline{C_{\mathrm{s}}}}^{-}+D^{\mathrm{lz}}{\overline{\left(\frac{\partial C_{1}}{\partial z}\right)}}^{+}-\bar{g}_{1}^{-} D^{\mathrm{mz}}{\overline{\left(\frac{\partial C_{1}}{\partial z}\right)}}^{-}$,

where $\epsilon_{\mathrm{ml}}=z^{+}-z^{-}$is the thickness of the control volume, $\langle C\rangle_{\mathrm{ml}}$ is the average solute concentration in the control volume, $\bar{\chi}^{+}$and $\bar{\chi}^{-}$signify the average of a quantity $\chi$ across the upper and lower volume boundary, respectively, and the subscript 's' stands for the solid phase. A few assumptions can be made to simplify this balance. If the lower bound of the control volume is sufficiently far within the mushy zone, it can be safely assumed that the average solute concentration in the liquid at that boundary is at local equilibrium, i.e. following Eq. (3). The phase field simulation, Fig. 3, illustrates this point quite clearly; the contour lines of composition are horizontal within the mushy zone, but not at the dendrite tips. It follows that $\bar{C}_{1}^{-}=C_{1}^{\mathrm{mz}}\left(z^{-}\right)$and ${\overline{\left(\partial C_{\mathrm{l}} / \partial z\right)}}^{-}=$ $G_{T} / m$. Within the mushy zone (lower bound), it is further assumed that the solute concentration in the solid can be approximated by the equilibrium solute 
concentration, i.e. $\bar{C}_{\mathrm{s}}^{-}=k \bar{C}_{1}^{-}$. Thus, Eq. (12) can be simplified to

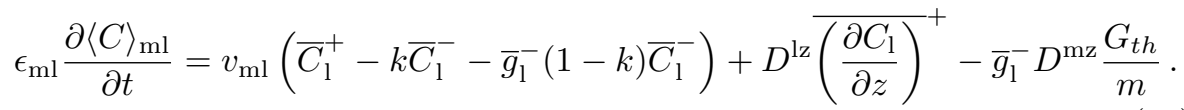

Two further assumptions are made that are more difficult to justify but that lead to a significant simplification of the model. First, it is assumed that the thickness of the boundary region, $\epsilon_{\mathrm{ml}}$, is small compared to the thickness of the mushy zone and that difference between the solute concentrations at the two bounds is therefore small. Thus, $C_{\mathrm{ml}} \approx \bar{C}_{1}^{+} \approx \bar{C}_{1}^{-}$. It is further assumed that the accumulation term in the balance (the l.h.s. term of Eq. (13)) is negligible. By introducing a representative liquid fraction for a sharp boundary, $g_{\mathrm{ml}}$, the control volume balance is transformed into a boundary condition,

$$
\left(1-g_{\mathrm{ml}}\right) \cdot(1-k) \cdot C_{\mathrm{ml}} \cdot v_{\mathrm{ml}}=g_{\mathrm{ml}} D^{\mathrm{mz}} \frac{G_{\mathrm{th}}}{m}-D^{\mathrm{lz}} G_{\mathrm{sol}} .
$$

The liquid fraction at the boundary is obtained from the solution of Eq. (5), i.e. $g_{\mathrm{ml}}=g_{1}\left(z_{\mathrm{ml}}\right)$, the solute concentration in the liquid is the equilibrium concentration, i.e. $C_{\mathrm{ml}}=C_{\mathrm{l}}^{\mathrm{mz}}\left(z_{\mathrm{ml}}\right)$, and $G_{\mathrm{sol}}$ is the solute gradient in the liquid zone just ahead of $z_{\mathrm{ml}}$. $G_{\mathrm{sol}}$ has a negative value. The same solute concentration is used as a boundary condition for the diffusion equation in the liquid zone, Eq. (8): $C_{1}^{\mathrm{lz}}\left(z_{\mathrm{ml}}\right)=C_{1}^{\mathrm{mz}}\left(z_{\mathrm{ml}}\right)$. The solution thus features continuity of the solute concentration in the liquid and a jump of the liquid fraction and of the solute concentration gradient at the mushy-liquid boundary. The validity of the different assumptions taken in deriving this interface balance will be verified through comparisons to the phase-field simulation of Boussinot [2], which provides detailed information on the local fields.

\subsection{Solution Methodology}

Equations (3) (7), (8), (10), and (14), together with the initial conditions in Eqs. (4) and (5) provide the complete problem statement to determine the evolution of a mushy zone within a static temperature gradient. The governing equations were discretized within MATLAB using a centred implicit scheme in both the liquid and mushy zones, and then resolved using the Gauss-Seidel calculation method. Each zone is discretized into 50 grid points. Unlike the models of Beckermann et al. [14, 18], which used a single-domain formulation and thus required a strong grid refinement at the interface in order to describe the jump between the mushy and liquid zones as a sharp transition of a continuous property, this model describes the interfaces as boundary conditions between the three subdomains. In this way, the jumps are easily accommodated and accurately described without the need of grid refinement.

To account for the motion of the interfaces $z_{\mathrm{ml}}$ and $z_{\mathrm{sc}}$, Landau transformations were applied giving two new position variables for the liquid and mushy zones, $\xi \in[0,1]$ and $\eta \in[0,1]$,

$$
\xi(z)=\frac{z-z_{\mathrm{ml}}}{H-z_{\mathrm{ml}}}
$$




$$
\eta(z)=\frac{z-z_{\mathrm{sc}}}{z_{\mathrm{ml}}-z_{\mathrm{sc}}}
$$

where $H$ is the total height of the domain. This enabled the system to be solved with fixed boundaries. The solute conservation equation in the liquid zone, Eq. (8), and the equation for the evolution of the liquid fraction in the mushy zone, Eq. (7), in the transformed coordinate system are

$$
\frac{\partial C_{\mathrm{l}}^{\mathrm{lz}}}{\partial t}=-v_{\mathrm{ml}} \cdot\left(\frac{\xi-1}{H-z_{\mathrm{ml}}}\right) \cdot \frac{\partial C_{\mathrm{l}}}{\partial \xi}+\frac{D^{\mathrm{lz}}}{\left(H-z_{\mathrm{ml}}\right)^{2}} \cdot \frac{\partial^{2} C_{\mathrm{l}}^{\mathrm{lz}}}{\partial \xi^{2}}
$$

and

$$
\frac{\partial g_{\mathrm{l}}}{\partial t}=\left(\frac{v_{\mathrm{sc}}+\eta\left(v_{\mathrm{ml}}-v_{\mathrm{sc}}\right)}{z_{\mathrm{ml}}-z_{\mathrm{sc}}}+\frac{D^{\mathrm{mz}} G_{t h}}{m(1-k) C_{\mathrm{l}}^{\mathrm{mz}}(z)\left(z_{\mathrm{ml}}-z_{\mathrm{sc}}\right)}\right) \frac{\partial g_{\mathrm{l}}}{\partial \eta}
$$

The solute balance at the mush-liquid interface, Eq. (14) is transformed to

$$
\left(1-g_{\mathrm{ml}}\right) \cdot(1-k) \cdot C_{\mathrm{ml}} \cdot v_{\mathrm{ml}}=g_{\mathrm{ml}} D^{\mathrm{mz}} \frac{G_{\mathrm{th}}}{m}-\frac{D^{\mathrm{lz}}}{H-z_{\mathrm{ml}}} \frac{\partial g_{\mathrm{l}}}{\partial \xi} .
$$

The equation for the solute concentration field in the liquid, within the mushy zone (Eq. (3)), and the expression for the velocity of the solid crust (Eq. (10)) remain the same using the transformations between the fixed and the Landau coordinates.

At each time-step, the solute concentration in the liquid zone and the liquid fraction in the mushy zone are calculated, and then the boundary conditions are applied to determine the evolution in $z_{\mathrm{sc}}$ and $z_{\mathrm{ml}}$.

\section{Validation by comparison to phase-field simulations}

\subsection{Application of volume averaging in a columnar mushy zone}

The volume average method is based on the concept of a representative elementary volume (REV). An REV must be sufficiently large to represent the averaged quantities of the local (microscopic) structure at the mesoscopic length scale, yet small enough such that important variations in the temperature, composition, and solid fraction can be captured over the entire (macroscopic) simulation domain. Fig. 5 provides a comprehensive analysis of the application of

the volume average formalism on a columnar mushy zone. Different sizes of $\mathrm{REV}$ are used to average the detailed microstructure that was obtained by the phase-field simulation in order to investigate the influence of the REV size on the representation of the mushy zone. In Fig. 5(a), the obtained variation in solid fraction as a function of height at the start of the holding stage is shown. A line average of the volume of the solid phase (i.e. an average made only across the width of the phase-field simulation domain), shown as a black line, represents the 1D distribution of the local solid fraction along the height of the mushy 
zone. Along with it, three coloured lines are shown that represent the solid fraction calculated by applying a centred moving average using averaging volumes of different height to the phase field data. As can be seen, the phase field result shows a non-monotonic decrease in solid fraction from the base of the domain to the dendrite tip. The maxima represent the secondary arms and the deep minima represent the interdendritic regions between the arms. The coloured lines demonstrate the volume average representation of the mushy zone; each averaged domain has approximately the same solid fraction as given by the phase field simulation, but all of the details representing the internal microstructure, i.e. the secondary arms and the interdendritic liquid, are not resolved. Far away from the dendrite tip, at high solid fraction, there is not much difference when using averaging volumes of different sizes, but the differences magnify as the dendrite tip is approached, because the macroscopic gradients become larger.

The variation in solid fraction with position obtained with the largest REV clearly shows that the average is not representative in the region where the length scale of the macroscopic variations is smaller than the REV size. Similarly, the solid fraction obtained with the smallest REV show wiggles that reflect local microstructural variations. Further, the location corresponding to the mushy-liquid boundary itself is at different positions depending on the size of the averaging volume, and none of them correspond to the phase field dendrite tip position. The mushy-liquid boundary consisting of dendrite tips represents a macroscopic discontinuity and thus cannot be averaged in a way that is independent of the size of the averaging volume. The average on both sides of such a macroscopic boundary is applicable only down to a distance of half the size of the REV from the boundary [19, 17, 20] (note that in general the sizes of the REV in the two macroscopic media can be different). As mentioned in Section 3.3, the boundary region itself represents a macroscopic discontinuity and must be treated separately.

Also shown in the figure is the analytically calculated solid fraction given by the Scheil equation, using the nominal solute concentration, $C_{0}$. The difference in the position of the mushy-liquid boundary is a consequence of the constitutional undercooling of the tip related to the dendrite tip growth kinetics. Using the Scheil equation, the mushy-liquid boundary is predicted to occur at a higher position (temperature) within the domain, corresponding to the phase diagram. The Scheil equation also gives a much higher solid fraction in the region behind the tip because of the assumption of infinitely fast diffusion in the liquid that does not account for the diffusion-controlled solidification kinetics. An additional contribution that lowers the solid fraction is the segregation of solute that transports solute by diffusion through the mushy zone towards the tips. In steady-state directional solidification, this segregation results in an increased average solute concentration $\left(C>C_{0}\right)$ behind the tips and thus delays solidification [21, 2]. The difference near the base of the mushy zone is due to the fact that the phase field simulation includes diffusion in the solid, while the Scheil equation does not [2].

Fig. 5(b) shows the variation in solid fraction at two positions within the phase field simulation domain as a function of the REV size. The locations of 


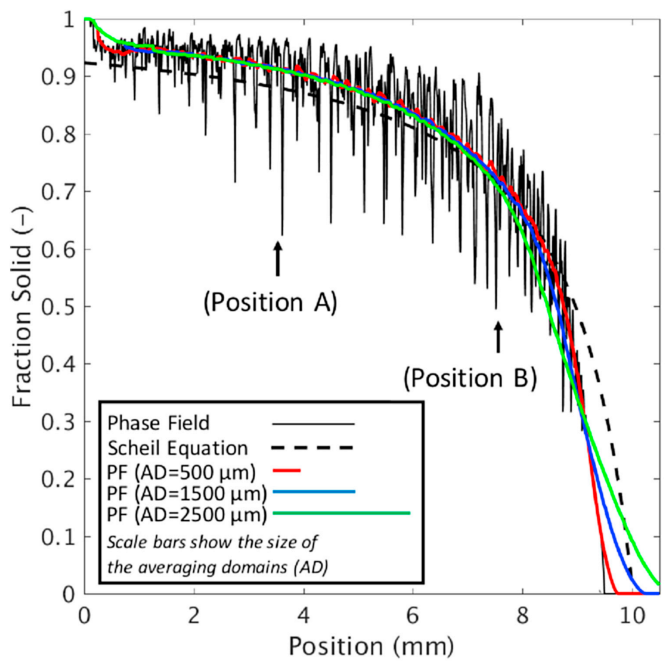

(a)

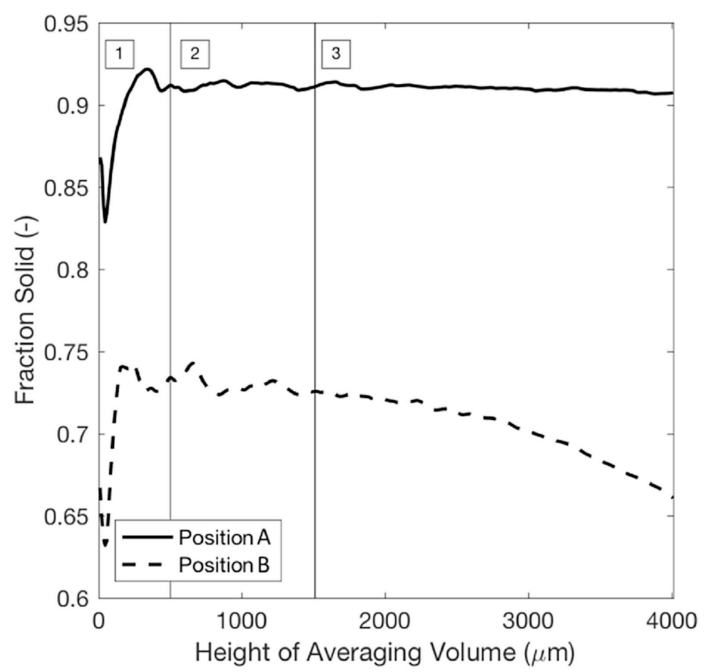

(b)

Figure 5: (a) Variation in the phase field predicted solid fraction as a function of length at the start of the hold $(t=0)$, along with the Scheil predictions based on the local solute composition. The coloured lines represent the average solid fraction for different moving average subvolumes; their length provides an indication of the difference in subvolume size. The markers (1), (2), and (3) refer to the Position 1 and Position 2 in (b), and the dendrite tip, respectively; (b) Variation in the phase field predicted solid fraction at two positions within the mushy zone as a function of averaging subvolume. The regions (1), (2), and (3) denote averaging volumes that are too small, ideal, and too large, respectively. 
these positions are identified in Fig. 5(a) by markers (A) and (B). Three different regimes can be identified. Regions 1 and 3 represents averaging volumes where the solid fraction changes significantly with increasing domain size, while Region 2 represents averaging volumes where there is only minimal change in solid fraction with varying domain size. In the case of Region 1, the averaging volume is too small to adequately smooth out local microstructural variations. Spatial variations in averaged fields that are defined by too small averaging volumes cannot be adequately described by volume average equations. In the case of Region 3, the averaging volume is too large to resolve the macroscale variations in solid fraction. The use of averaging volumes that are too large would thus lead to a loss of macroscopic information. Region 2 represents the adequate range of averaging volumes. The minimum averaging volume in the initial state of the mushy zone thus is $500 \mu \mathrm{m}$, which corresponds to approximately twice the secondary dendrite arm spacing $\left(\lambda_{2}\right)$ seen in the phase field simulation [2]. Thus, $2 \cdot \lambda_{2}$ is proposed as the ideal cell size for volume average methods.

\subsection{Comparison between the volume average model and the phase field simula- tion}

A validation of the volume average model is important because the simplifying assumptions taken to describe the mushy-liquid boundary may critically affect the prediction of the mushy zone dynamics. The phase field simulation of solidification in a static temperature gradient [2] can be used to validate the proposed volume average approach to this problem. The main focus of the validation is to ensure that the motion of $z_{\mathrm{ml}}$ is correct; the application of the volume average model deep in the mushy zone is rather trivial.

The process parameters and material properties used in the volume average model were identical to those used in the phase field simulation. The only exception were the diffusion coefficients of the solute in the mushy and liquid zones. In the volume average model they were assumed to be $4.00 \times 10^{-9} \mathrm{~m}^{2} / \mathrm{s}$, and $6.23 \times 10^{-9} \mathrm{~m}^{2} / \mathrm{s}$, respectively, instead of the temperature dependent values used in the phase field simulations. These diffusion coefficients were calculated based on the Arrhenius law provided in [2] using the median temperatures of each zone. All of the other required model inputs, including the initial position of the dendrite tips, $z_{\mathrm{ml}}^{0}$, and tip undercooling, $\Delta T_{c}$, were also taken from [2], and shown in Table 1. The initial conditions were determined as stated in Section 3.1. The mushy and liquid domains were discretized using a fixed grid size of $\Delta \epsilon=\Delta \eta=0.02$ in the Landau transformed coordinates, and a time step of $0.1 \mathrm{~s}$ was applied. The simulation time was negligible, on the order of 5 minutes

Fig. 6 shows the evolution of $z_{\mathrm{ml}}$ for both the volume average model and the phase field simulation in order to compare the overall system dynamics. Given the simplifications in the description of the mushy-liquid boundary, Eq. (13), it is quite impressive that the $z_{\mathrm{ml}}$ curve from the volume average model follows the same shape as in the phase field simulation. That is, an initial increase in $z_{\mathrm{ml}}$ because of the inertia effect due to the sudden freezing of the thermal gradient followed by a decrease in $z_{\mathrm{ml}}$ until a minimum point is reached and 
Table 1: Thermophysical properties and solidification parameters used in the volume average simulations.

\begin{tabular}{lrr}
\hline & $\begin{array}{r}\text { Comparison } \\
\text { with phase-field }\end{array}$ & $\begin{array}{r}\text { ESRF } \\
\text { experiment }\end{array}$ \\
\hline Bulk copper concentration, $C_{0}(\mathrm{wt} . \%)$ & 4 & 5 \\
Partition coefficient, $k(-)$ & 0.17 & 0.10 \\
Liquidus slope, $m(\mathrm{~K} / \mathrm{wt} . \%)$ & -3.37 & -2.70 \\
Melting point of pure Al, $T_{\mathrm{m}}(\mathrm{K})$ & 933.45 & 933.45 \\
Diffusion coefficient in the liquid zone, $D^{\mathrm{lz}}\left(\mathrm{m}^{2} / \mathrm{s}\right)$ & $6.23 \cdot 10^{-9}$ & $9.30 \cdot 10^{-9}$ \\
Diffusion coefficient in the mushy zone, $D^{\mathrm{mz}}\left(\mathrm{m}^{2} / \mathrm{s}\right)$ & $4.00 \cdot 10^{-9}$ & $3.10 \cdot 10^{-9}$ \\
Temperature gradient, $G_{\text {th }}(\mathrm{K} / \mathrm{m})$ & 10000 & 2700 \\
Initial velocity of the dendrites, $V_{\text {tip }}(\mathrm{m} / \mathrm{s})$ & $6.4 \cdot 10^{-6}$ & $6.4 \cdot 10^{-6}$ \\
Initial position of the mushy-liquid interface, $z_{\mathrm{ml}}^{0}(\mathrm{~m})$ & $9.49 \cdot 10^{-3}$ & $5.00 \cdot 10^{-3}$ \\
Initial position of the solid-mushy interface, $z^{0}(\mathrm{~m})$ & 0 & 0 \\
Dendrite tip constitutional undercooling, $\Delta T_{c}(\mathrm{~K})$ & 5.20 & 0.89 \\
Total height of the domain $(\mathrm{m})$ & $3.15 \cdot 10^{-2}$ & $3.70 \cdot 10^{-2}$ \\
\hline
\end{tabular}

then a slow advance. The difference between the two curves is less than $2 \%$ of the length of the mushy zone, although it would seem that the velocity of the mushy-liquid boundary, $v_{\mathrm{ml}}$, is greater in the volume average model than the phase field simulation when $t>1000 \mathrm{~s}$. This is evident from the fact that the two curves are diverging.

Fig. 7 compares several physical quantities between the two simulations: (a) the solute composition in the liquid at $z_{\mathrm{ml}}$, (b) the average composition gradient in the liquid just ahead of $z_{\mathrm{ml}}$, and (c) the liquid fraction in the mushy zone at $z_{\mathrm{ml}}$. For (a) and (c), the averaging volume of $500 \mu \mathrm{m} \approx 2 \cdot \lambda_{2}$ applied to the phase field result was situated entirely within the mushy zone as indicated by the dotted box in the inset images of (a). The average composition gradient was calculated by applying a central-difference first derivative, centred at one grid distance ahead of $z_{\mathrm{ml}}$ to the line average across the width of the phase field domain. In all of these figures, the results obtained from the volume average model match closely to the phase field results, although they are not identical. Three additional lines are plotted on Fig. Z7(a). These lines correspond to $C_{1}^{*}$ calculated from the phase diagram based on temperatures at the bottom, middle, and top of the averaging volume associated with the curve "Phase Field (500 $\mu \mathrm{m}$ avg domain)". As can be seen, the average phase field solute composition in the liquid at $z_{\mathrm{ml}}$ matches quite well against the equilibrium solute concentration at the mid-height of the averaging volume, especially at longer times. The initial difference between these two curves is due to the fact that the region near the dendrite tips in the phase field simulation is initially outof-equilibrium with the thermal fields whereas complete equilibrium is assumed to always exist in the volume average simulation. As can be seen in the inset contour plots showing some phase field results, the solute contours at $t=0$ follow the shape of the dendrites and thus the average value is greater than the 


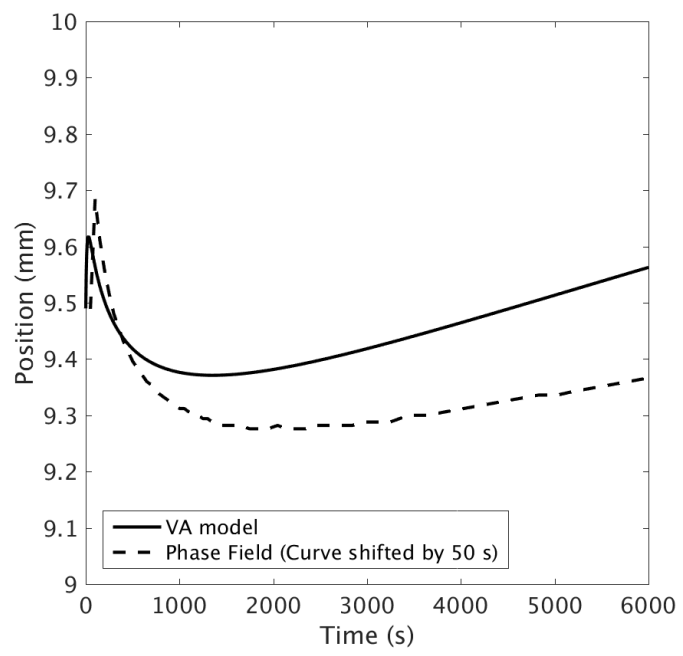

Figure 6: Comparison of the evolution in $z_{\mathrm{ml}}$ between the volume average model and the phase field simulation. Note that the volume average result is shifted by $50 \mathrm{~s}$ to the right in order to improve the figure clarity.

equilibrium value at mid-height. As time advances, the lateral solute concentration gradients diminish and the solute concentration in the liquid approaches the equilibrium solute concentration, resulting in a match between these two curves. The volume average model prediction of the solute composition in the liquid at $z_{\mathrm{ml}}$ is seen to be quite lower than the average value from the phase field simulation, and is near the equilibrium solute concentration found at the top of the averaging volume. This is because the $z_{\mathrm{ml}}$ predicted by the volume average model is at a higher position and thus at a higher temperature within the domain as compared to the phase field result.

The results shown in Fig. 7 demonstrate clearly that the volume average method can be successfully applied to simulate solidification within a static temperature gradient at high thermal gradients, as well as simulate the evolution of the mushy-liquid interface, even though the microstructure within the mushy zone substantially changes over time starting from a parabolic dendrite tip evolving towards a rather planar front with liquid channel (Fig. 7 (a)). The volume average model does not account for this internal morphology changes but obviously captures the major contributions to the average front movement. These figures can also be used to explain the similarities and differences in $z_{\mathrm{ml}}$ between the two curves seen in Fig. 6. First, by analyzing the boundary condition at $z_{\mathrm{ml}}$, Eq. (14), it can be seen that the sign of the velocity $v_{\mathrm{ml}}$ is determined by the difference between the two fluxes on the r.h.s. of the equation, i.e. $v_{\mathrm{ml}}>0$ when $\left(m G_{s o l} / g_{\mathrm{ml}} G_{t h}\right)>1$, and vice versa. Second, the magnitude of the velocity $v_{\mathrm{ml}}$ is given by $C_{\mathrm{ml}}$ and $g_{\mathrm{ml}}$; specifically $v_{\mathrm{ml}}$ is equal to the flux difference divided by $(1-k)\left(1-g_{\mathrm{ml}}\right) C_{\mathrm{ml}}$. Examining Fig. Z (a) and (c), it can 


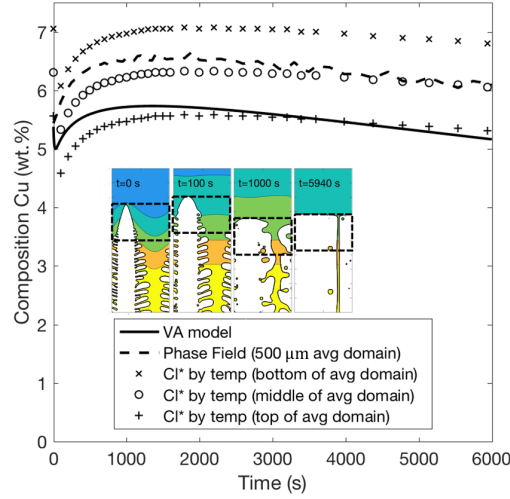

(a)

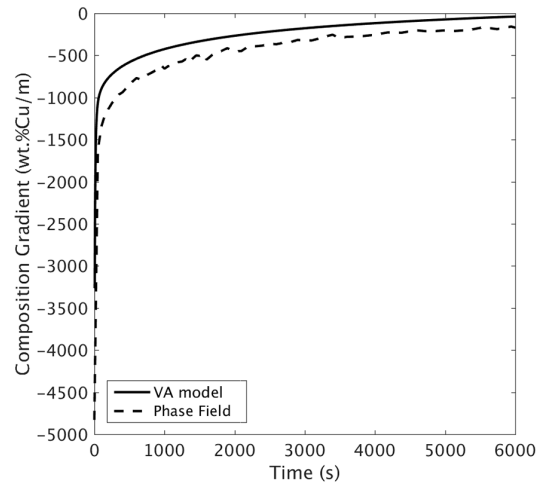

(b)

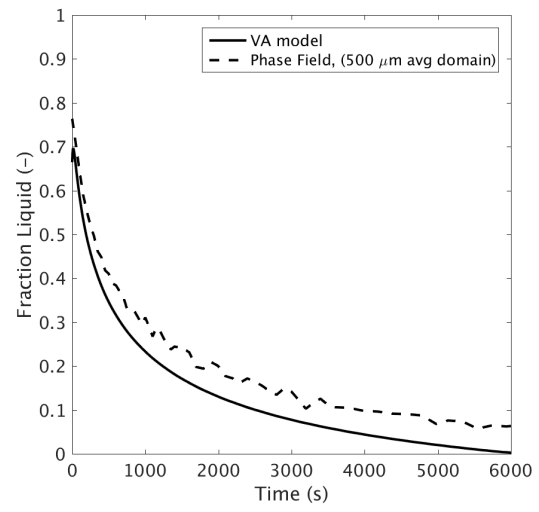

(c)

Figure 7: Comparison of the evolution in (a) $C_{1}^{*}$, (b) $\frac{\partial C_{1}}{\partial z}$, and (c) $f_{1}$ at $z_{\mathrm{ml}}$ between the volume average model and the phase field simulation. The inset contour plots in (a) show the out-of-equilibrium solute field in the liquid relative to the thermal gradient; the dotted-line boxes identify the averaging domain. 
be seen that while the difference in $C_{\mathrm{ml}}$ between the volume average model and the phase field simulation is of the order of $20 \%$ during the whole evolution, the difference in $g_{\mathrm{ml}}$ increases with time and becomes larger than $50 \%$ at $t=3500 \mathrm{~s}$. Since $\left(1-g_{\mathrm{ml}}\right)$ is thus too large, and $C_{\mathrm{ml}}$ is too small in the volume average model as compared to the phase field simulation, they seem to compensate each other. The error in the magnitude of the solute concentration gradient in the liquid, Fig. 7(b) follows a similar trend to $g_{\mathrm{ml}}$ both in magnitude and in sign. Thus, as compared to the phase field simulation, both terms on the r.h.s. of the simplified solute balance, Eq. (13), are too small in the volume average model. Specifically, the solute flux on the liquid side, which pulls $z_{\mathrm{ml}}$ to advance, is weaker than in the phase field simulation because of the error in the solute concentration gradient, while the solute flux on the mushy side, which pulls $z_{\mathrm{ml}}$ to retreat, is weaker because of the error in $g_{\mathrm{ml}}$. Nevertheless, as it is the difference of these two fluxes that results in the observed evolution in $z_{\mathrm{ml}}$, a strong match between the volume average model and the phase field simulation is achieved.

\section{Comparison between the volume average model and the ESRF experiments}

The comparison between the volume average model and phase field simulation validated the use of a volume averaging method for simulating mushy zone dynamics within a static temperature gradient. A comparison against the experimental data [1] is now performed in order to demonstrate that this approach properly describes the observed phenomena. For this comparison, the diffusion coefficient of the solute in the mushy zone was taken from [1]. The mushy and liquid zones were again discretized using a fixed grid size of $\Delta \epsilon=\Delta \eta=0.02$ in the Landau transformed coordinates and a time step of $0.1 \mathrm{~s}$ was applied. $\Delta T_{c}=0.89^{\circ} \mathrm{C}$ and $C_{l}^{0}\left(z_{\mathrm{ml}}^{0}\right)=5.32 \mathrm{wt} . \mathrm{Cu} \%$ were determined using the KGT model from the dendrite tip velocity measured in the ESRF experiment at the end of the cooling stage (which corresponds to the beginning of the holding stage) [1]. All of the other input parameters, including $z_{\mathrm{ml}}^{0}$ were taken from [1], and shown in Table 1.

Fig. 8 compares the evolution in $z_{\mathrm{ml}}$ and $z_{\mathrm{sc}}$ between the ESRF experiments and the volume average simulations. First, as can be seen, the volume average predictions of $z_{\mathrm{ml}}$ are strongly linked to the the diffusion coefficient. When $D^{\mathrm{lz}}=$ $D^{\mathrm{mz}}$, the retreat of $z_{\mathrm{ml}}$ occurs relatively quickly, and the planar interface (i.e. $\left.z_{\mathrm{sc}}=z_{\mathrm{ml}}\right)$ is achieved in the simulation far earlier than the ESRF experiment. However, an increase in $D^{\mathrm{lz}}$ to $3 \cdot D^{\mathrm{mz}}$ results in excellent agreement between the simulation and experiment. Theoretically, $D^{\mathrm{mz}}$ and $D^{\mathrm{lz}}$ should be quite similar, since the thermal gradient is relatively low. The need to increase $D^{\mathrm{lz}}$ by a significant amount provides strong indication that, in addition to diffusion, solute convection is occurring within the liquid zone of the ESRF experiment. An indication of convection currents was already observed in the experimental images from the curvature within the columnar front and the presence of liquid channels along the sides of the sample [1, 22]. Second, the volume average

predictions of $z_{\mathrm{sc}}$ match closely the ESRF experiment, and are independent of 


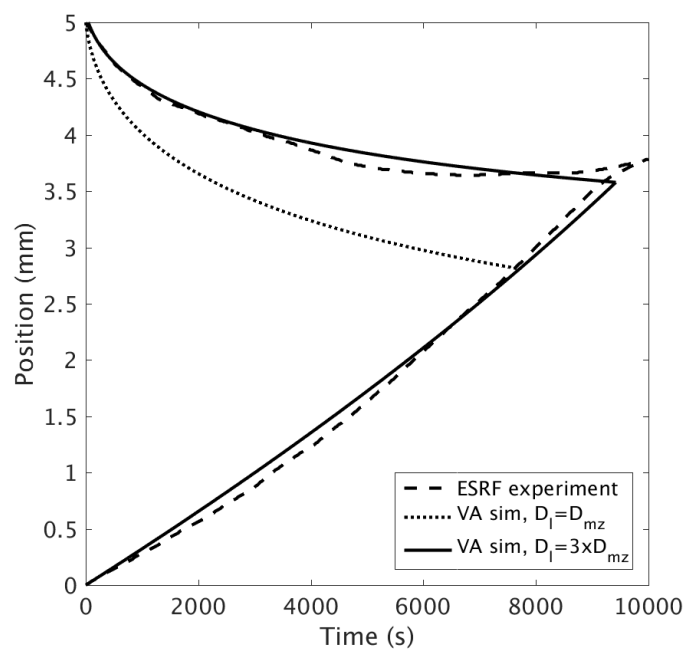

Figure 8: Comparison of the evolution in $z_{\mathrm{sc}}$ and $z_{\mathrm{ml}}$ between the volume average model (using two different values for $D^{\mathrm{lz}}$ ) and the ESRF X-ray radiography experiment. The curves beginning at $0 \mathrm{~mm}$ and $5 \mathrm{~mm}$ correspond to $z_{\mathrm{sc}}$ and $\mathrm{z}_{\mathrm{ml}}$ respectively. Note that two VA curves for $z_{\mathrm{sc}}\left(D_{l}=D_{m z}\right.$ and $\left.D_{l}=3 D_{m z}\right)$ overlap.

$D^{\mathrm{lz}}$. This is not surprising, given that the motion of $z_{\mathrm{sc}}$ is governed only by $D^{\mathrm{mz}}$ as shown in Eq. 10. The final time for the transition to a planar interfact found using the volume average model was 9420 s, whereas in the ESRF experiment it was $9815 \mathrm{~s}$. Given all the assumptions inherent in the volume average method, the similarity is excellent.

Fig. 9 compares several physical quantities at mushy-liquid boundary predicted by the volume average model and measured in the experimental data: (a) solute composition in the liquid at $z_{\mathrm{ml}}$, (b) diffusive flux in the liquid ahead of $z_{\mathrm{ml}}$, and (c) the liquid fraction in the mushy zone at $z_{\mathrm{ml}}$. The measurements were performed using X-ray radiographic image processing methods as detailed in Section 2.3 of [1]. Note, that in Fig. 9(b), the diffusive flux was plotted in the figure and not the solute concentration gradient (Fig. 6(b)) because of the artificial enhancement of the liquid diffusion coefficient in the volume average model. In all cases, given the model assumptions and complexity in experimental measurements, the results between the VA model and experimental observations are seen to be quite similar. As seen in Fig. 9 (b) and (c), the calculations of the evolution in the diffusive flux in the liquid ahead of the mushy zone and the liquid fraction at the interface accurately describe the experimental observations. Although the calculations of the solute concentration evolution at $z_{\mathrm{ml}}$ do not exactly fit the experimental measurements, Fig. 9(a), the observed difference is similar to the difference seen in Fig. 6 (a) in both magnitude and sign.

The results shown in Figs. 8 and 9 demonstrate that while the volume average method is able to describe the observed phenomena when solidification is oc- 


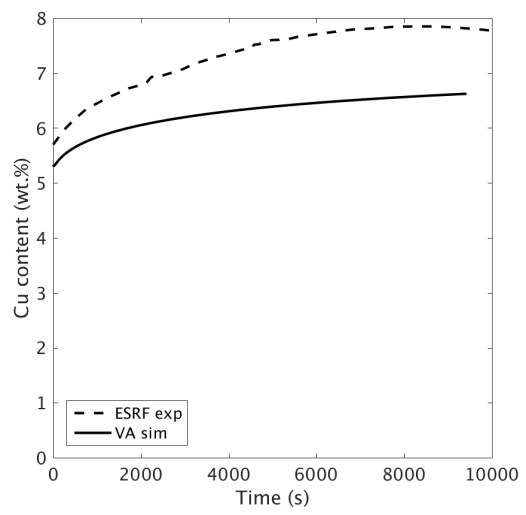

(a)

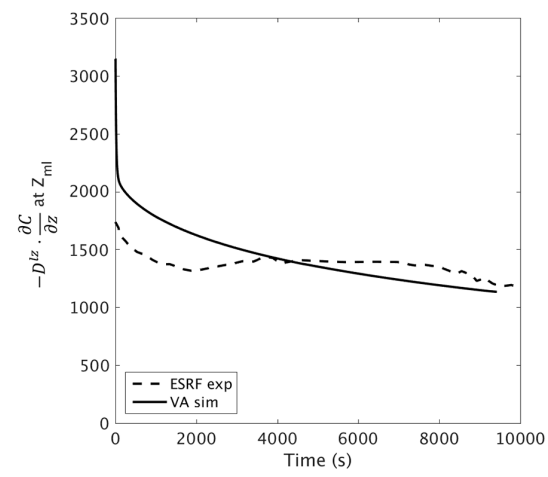

(b)

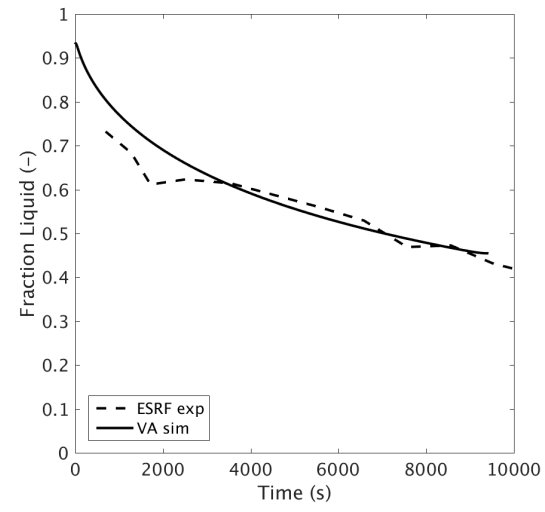

(c)

Figure 9: Comparison of the evolution in (a) $C_{1}^{*}$, (b) $-D \cdot \frac{\partial C_{1}}{\partial z}$, and (c) $f_{1}$ at $z_{\mathrm{ml}}$ between the volume average model assuming $D^{\mathrm{lz}}=3 \cdot D^{\mathrm{mz}}$, and the ESRF X-ray radiography experiment. 
curring within a static gradient, it is necessary to account for convection within the liquid. This allows for greater understanding of solidification phenomena occurring during X-ray radiographic imaging. However, accurate knowledge of the initial alloy composition is needed to understand the additional observed discrepancies.

\section{Conclusions}

A volume average model to study the transition of a semi-solid mushy zone to a planar interface in a static temperature gradient is proposed. This model does not directly describe microscopic phenomena such as liquid film migration, coarsening, thermo-solutal migration, TGZM, and microsegregation patterns. Instead, it provides a volume average description of the principal phenomena governing the mushy zone dynamics, including solute diffusion in the interdendritic and bulk liquids, the migration of the solid-liquid interface at the bottom of the mushy zone, the motion of the boundary between the mushy and bulk liquid zones, and the liquid-to-solid phase transformation.

A significant effort has been applied to properly describe the boundary between the mushy and liquid zones. It has been shown that a standard volume average description is not applicable to its treatment. Instead, a special control volume enclosing the boundary must be used in order to introduce the mass transfer balance that determines its motion. Using simplifying assumptions related to solute diffusion at the microscale, these balance equations can be reduced to a boundary condition. A phase-field simulation providing detailed data on the dendrite morphologies and on the solute fields in the liquid near the dendrites was used to verify the simplifying assumptions to the mushy-liquid boundary. Although a certain error is introduced by the simplifications, the interface dynamics can nonetheless be accurately predicted.

The new volume average model has been validated by comparison against both in-situ experimental observations and phase-field simulations of the mushyto-planar transition in an $\mathrm{Al}-\mathrm{Cu}$ alloy. A very good prediction of the observed experimental and phase-field dynamics was achieved with this new model even though the described system was only one-dimensional. However, an augmentation of the solute diffusion coefficient in the bulk liquid was required in order to mimic the convective solute transport present in the in situ experiment. This new volume average model provides a more accurate description of the mushy zone dynamics as compared to previous models by taking into account solute partitioning and the motion of the two boundaries between the solid, mushy, and liquid zones. Further, the need to carefully describe the mushy-liquid boundary when using volume average approaches to model solidification mechanisms is demonstrated.

\section{Acknowledgements}

This research was supported by the French State through the program "Investment in the future" operated by the National Research Agency (ANR) and 
referenced by ANR-11 LABX-0008-01 (LabEx DAMAS). G.B. and M.A. acknowledge financial support from the German Research Foundation (DFG) in the context of the Collaborative Research Centre SFB1120 "Precision Melt Engineering". H.N.-T. and G.R. acknowledge financial support from the XRMON project (AO-2004-046) of the MAP programme of the European Space Agency (ESA) and from the French National Space Agency (CNES).

\section{References}

[1] G. Salloum-Abou-Jaoude, G. Reinhart, H. Combeau, M. Založnik, T. Lafford, H. Nguyen-Thi, Quantitative analysis by in situ synchrotron X-ray radiography of the evolution of the mushy zone in a fixed temperature gradient, Journal of Crystal Growth 411 (2015) 88-95.

[2] G. Boussinot, M. Apel, Phase field and analytical study of mushy zone solidification in a static thermal gradient: From dendrites to planar front, Acta Materialia 122 (2017) 310-321.

[3] B.-O. Demory, M. Gillon, J. de Wit, N. Madhusudhan, E. Bolmont, K. Heng, T. Kataria, N. Lewis, R. Hu, J. Krick, V. Stamenkovic, B. Benneke, S. Kane, D. Queloz, A map of the large day-night temperature gradient of a super-Earth exoplanet, Nature 532 (7598) (2016) 207.

[4] M. Worster, H. Huppert, R. Sparks, The Crystallization of Lava Lakes, Journal of Geophysical Research 98 (B9) (1993) 15891-15901.

[5] H. Combeau, B. Appolaire, J.-M. Seiler, Interface temperature between solid and liquid corium in severe accident situations: A comprehensive study of characteristic time delay needed for reaching liquidus temperature, Nuclear Engineering and Design 240 (8) (2010) 1975-1985.

[6] J.-M. S. Mathiew Guillaumé, Hervé Combeau, An improved interface model for molten corium-concrete interaction, Nuclear Engineering and Design 239 (2009) 1084-1094.

[7] W. Pfann, Temperature gradient zone melting, Transactions of the AIME 203 (1955) 961-964.

[8] W. Tiller, Migration of a liquid zone through a solid (part 1), Journal of Applied Physics 34 (1963) 2757-2762.

[9] H. Nguyen-Thi, G. Reinhart, A. Buffet, T. Schenk, N. Mangelinck-Noël, H. Jung, N. Bergeon, B. Billia, J. Härtwig, J. Baruchel, In situ and real-time analysis of TGZM phenomena by synchrotron X-ray radiography, Journal of Crystal Growth 310 (11) (2008) 2906-2914.

[10] S. Fischer, M. Založnik, J.-M. Seiler, M. Rettenmayr, H. Combeau, Experimental verification of a model on melting and resolidification in a temperature gradient, Journal of Alloys and Compounds 540 (2012) 85-88. 
[11] D. Liu, Y. Su, X. Li, L. Luo, J. Guo, H. Fu, Influence of thermal stabilization on the solute concentration of the melt in directional solidification, Journal of Crystal Growth 312 (2010) 3658-3664.

[12] T. Liu, L. Luo, L. Wang, N. Guo, X. Li, R. Chen, Y. Su, J. G. Guo, H. Fu, Influence of thermal stabilization treatment on microstructure evolution of the mushy zone and subsequent directional solidification in ti-43al-3si alloy, Materials and Design 97 (2016) 392-399.

[13] N. R. Gewecke, T. P. Schulze, The rapid advance and slow retreat of a mushy zone, Journal of Fluid Mechanics 674 (2011) 1-17.

[14] C. Y. Wang, C. Beckermann, A multiphase solute diffusion model for dendritic alloy solidification, Metallurgical Transactions A 24 (December) (1993) 2787-2802. doi:10.1007/BF02659502.

[15] G. Salloum-Abou-Jaoude, In situ investigation by X-ray radiography of microstructure evolution during solidification of binary alloys, $\mathrm{PhD}$ thesis, Université d'Aix-Marseille (2014).

[16] W. Kurz, B. Giovanola, R. Trivedi, Theory of microstructural development during rapid solidification, Acta Metallurgica 34 (5) (1986) 823-830.

[17] S. M. Hassanizadeh, W. G. Gray, Derivation of conditions describing transport across zones of reduced dynamics within multiphase systems, Water Resources Research 25 (3) (1989) 529-539.

[18] M. A. Martorano, C. Beckermann, C.-A. Gandin, A solutal interaction mechanism for the columnar-to-equiaxed transition in alloy solidification, Metallurgical and Materials Transactions A 35 (6) (2004) 1915-1915. doi: 10.1007/s11661-004-0101-0.

[19] M. Hassanizadeh, W. G. Gray, General conservation equations for multiphase systems: 1. Averaging procedure, Advances in Water Resources 2 (C) (1979) 131-144.

[20] J. C. Parker, M. T. van Genuchten, Flux-Averaged and Volume-Averaged Concentrations in Continuum Approaches to Solute Transport, Water Resources Research 20 (7) (1984) 866-872.

[21] A. Viardin, M. Založnik, Y. Souhar, M. Apel, H. Combeau, Mesoscopic modeling of spacing and grain selection in columnar dendritic solidification: envelope versus phase-field model, Acta Materialia 122 (2017) 386-399.

[22] G. Reinhart, C.-A. Gandin, N. Mangelinck-Noël, H. Nguven-thi, J.-E. Spinelli, J. Baruchel, B. Billia, Influence of natural convection during upward directional solidification: A comparison between in situ X-ray radiography and direct simulation of the grain structure, Acta Materialia 61 (13) (2013) 4765-4777. doi:10.1016/j .actamat.2013.04.067.

URL http://linkinghub.elsevier.com/retrieve/pii/ S135964541300356X 